\title{
SCIENTIFIC REPORTS

\section{OPEN A Comparison Study of Vitamin D Deficiency among Older Adults in China and the United States}

\begin{abstract}
Jia Wei ${ }^{1,3}$, Anna Zhu ${ }^{1,3}$ \& John S. Ji $\mathbb{1}^{1,2^{*}}$
Vitamin $D$ deficiency is a common health concern worldwide. We aim to compare the prevalence of vitamin D deficiency among older adults (65+) in China and the United States (US). We used data from the 2011 wave of Chinese Longitudinal Healthy Longevity Survey (CLHLS) in China $(n=2180)$, and 2011-2014 National Health and Nutrition Examination Survey (NHANES) in the US $(n=2283)$. Serum 25 -hydroxyvitamin $D[25(\mathrm{OH}) \mathrm{D}]$ was measured and a level of under $30 / 50 \mathrm{nmol} / \mathrm{L}$ was defined as vitamin $D$ severe deficiency/deficiency. Risk factors of vitamin $D$ deficiency were examined by multivariate regression models. We found that the mean $25(\mathrm{OH}) \mathrm{D}$ concentration was lower in China than in the US ( 45.1 vs. $83.5 \mathrm{nmol} / \mathrm{L}$ ), with Chinese elderly lower than American elderly for every age group. $70.3 \%$ in China and $17.4 \%$ in the US were considered as vitamin D deficiency $(30.6 \%$ and $3.4 \%$ were considered as severe deficiency). Older age, females, ethnic minorities, higher household income, self-rated "very bad" health, and never drinkers, were statistically significant in predicting lower serum 25(OH)D levels in China. In the US, males, ethnic minorities, lower income, self-rated "very bad" health, physically inactive, overweight, and obese were related to lower serum 25(OH)D levels. Our findings suggest that different interventional strategies are needed to improve vitamin $D$ deficiency and its associated negative health outcomes in China and the US.
\end{abstract}

Vitamin D deficiency is a serious health condition worldwide. Vitamin D is essential for human bone health, and severe vitamin D deficiency increases the risk of many diseasesincluding osteomalacia, osteoporosis, muscle weakness, hip fractures, diabetes, cancer, heart disease, arthritis, and poor general health in the elderly ${ }^{1-3}$. The aging process is considered a risk of vitamin D deficiency, because of debilitated ability to synthesize vitamin D from sunlight, activation of vitamin D in the kidney, and less outdoor exercise and activity ${ }^{4}$.

The US National Academy of Medicine (formerly the Institute of Medicine) considers a serum 25-hydroxyvitamin $\mathrm{D}(25(\mathrm{OH}) \mathrm{D})$ level of at least $50 \mathrm{nmol} / \mathrm{L}$ as the adequate exposure to vitamin $\mathrm{D}$ to maintain bone health. Individuals with levels less than $30 \mathrm{nmol} / \mathrm{L}$ are considered as severe deficient ${ }^{5}$. In this study, we aim to report serum $25(\mathrm{OH}) \mathrm{D}$ concentrations in China and the United States (US) using data from the CLHLS (Chinese Longitudinal Healthy Longevity Survey) and the NHANES (US National Health and Nutrition Examination Survey), which are nationally representative surveys of older adults from both countries.

\section{Results}

CLHLS had a larger proportion of older adults aged 80 years and older (67.0\%), with a mean age of 85.9 years old, compared to $26.7 \%$ in NHANES with a mean age of 73.3 years old (Table 1 ). In China, only $37.0 \%$ of the participants had some formal education, while in the US, around $70 \%$ had a high school education and above. More Chinese participants were widowed (57.4\%) than US participants (26.8\%). More Chinese participants rated their health condition as "good" (35.5\%) than US participants (23.1\%), and fewer rated as "bad" in China (11.2\%) than in the US (23.7\%). Smoking and drinking behaviors were more common in the US than in China. More Chinese participants never smoked (72.6\%) or drank any alcohol (76.6\%) than US participants (50.0\% and $18.0 \%$, respectively). The Chinese sample was more physically inactive than the US sample $(80.3 \%$ versus $41.2 \%$ do not have physical activity). China had much more underweight participants (24.0\%) than the US (1.7\%), while the US had much more overweight and obese participants ( $35.4 \%$ and $34.1 \%$, respectively) than China ( $10.6 \%$ and $3.1 \%$, respectively).

${ }^{1}$ Environmental Research Center, Duke Kunshan University, Kunshan, Jiangsu, China. ${ }^{2}$ Nicholas School of the Environment, Duke University, Durham, North Carolina, USA. ${ }^{3}$ These authors contributed equally: Jia Wei and Anna Zhu.*email: john.ji@dukekunshan.edu.cn 


\begin{tabular}{|c|c|c|c|}
\hline \multicolumn{2}{|l|}{ China (CLHLS 2011) } & \multicolumn{2}{|l|}{ US (NHANES 2011-2014) } \\
\hline & $\mathbf{n}, \%$ & & $\mathbf{n}, \%$ \\
\hline Total & $2,180(100)$ & Total & $2,283(100)$ \\
\hline Age $($ mean \pm SD) & $85.9 \pm 12.0$ & Age $($ mean \pm SE $)$ & $73.3 \pm 0.1$ \\
\hline Age group & & Age group & \\
\hline $65-69$ & $247(11.3)$ & $65-69$ & $703(30.8)$ \\
\hline $70-74$ & $249(11.4)$ & $70-74$ & $592(25.9)$ \\
\hline $75-79$ & $223(10.2)$ & $75-79$ & $379(16.6)$ \\
\hline $80+$ & $1,461(67.0)$ & $80+$ & $609(26.7)$ \\
\hline Gender & & Gender & \\
\hline Male & $991(45.5)$ & Male & $1,111(48.6)$ \\
\hline Female & $1,189(54.5)$ & Female & $1,172(51.4)$ \\
\hline Race & & Race/Ethnicity & \\
\hline Han Chinese & $1,970(90.4)$ & Mexican American & $174(7.7)$ \\
\hline Ethnic minorities & $158(7.3)$ & Other Hispanics & $197(8.6)$ \\
\hline \multirow[t]{4}{*}{ Missing } & $52(2.4)$ & Non-Hispanic White & $1,210(53.0)$ \\
\hline & & Non-Hispanic Black & $464(20.3)$ \\
\hline & & Non-Hispanic Asian & $202(8.9)$ \\
\hline & & Other races & $36(1.6)$ \\
\hline Education & & Education & \\
\hline No formal education & $1,353(62.1)$ & Less than 9th grade & 353 (15.5) \\
\hline Formal education & $806(37.0)$ & 9-11th grade (Includes 12th grade with no diploma) & $329(14.4)$ \\
\hline \multirow[t]{4}{*}{ Missing } & $21(1.0)$ & High school graduate/GED or equivalent & $528(23.1)$ \\
\hline & & Some college or AA degree & $580(25.4)$ \\
\hline & & College graduate or above & $488(21.4)$ \\
\hline & & Missing & $5(0.2)$ \\
\hline Marital Status & & Marital Status & \\
\hline Married & $804(36.9)$ & Married & $1,250(54.8)$ \\
\hline Separated & $39(1.8)$ & Separated & $48(2.1)$ \\
\hline Divorced & $5(0.2)$ & Divorced & $270(11.8)$ \\
\hline Widowed & $1,252(57.4)$ & Widowed & $613(26.8)$ \\
\hline Never married & $23(1.1)$ & Never married & $100(4.4)$ \\
\hline Missing & $57(2.6)$ & Missing & $2(0.1)$ \\
\hline Household income & & Income(PIR) & \\
\hline Tertile1 (0-6,000 RMB) & $719(33.0)$ & $0-1.85$ & $974(42.7)$ \\
\hline Tertile2 (6,200-20,000 RMB) & $698(32.0)$ & $1.86-3.50$ & $550(24.1)$ \\
\hline $\begin{array}{l}\text { Tertile3 (21,000-more than } \\
100,000 \text { RMB) }\end{array}$ & $623(28.6)$ & $>3.51$ & $558(24.4)$ \\
\hline Missing & $140(6.4)$ & Missing & $201(8.8)$ \\
\hline Health condition & & Health condition & \\
\hline Very good & $106(4.9)$ & Very good & $160(7.0)$ \\
\hline Good & $774(35.5)$ & Good & $527(23.1)$ \\
\hline Fair & $839(38.5)$ & Fair & $840(36.8)$ \\
\hline Bad & $243(11.2)$ & Bad & $540(23.7)$ \\
\hline Very Bad & $18(0.8)$ & Very Bad & $104(4.5)$ \\
\hline Missing & $200(9.2)$ & Missing & $112(4.9)$ \\
\hline Smoking status & & Smoking status & \\
\hline Never smoker & $1,582(72.6)$ & Never smoker & $1,142(50.0)$ \\
\hline Former smoker & $176(8.1)$ & Past smoker & $908(39.8)$ \\
\hline Current smoker & $356(16.3)$ & Current smoker & $231(10.1)$ \\
\hline Missing & $66(3.0)$ & Missing & $2(0.1)$ \\
\hline Drinking status & & Drinking status & \\
\hline Never drinker & $1,670(76.6)$ & Never drinker & $411(18.0)$ \\
\hline Former drinker & $128(5.9)$ & Past drinker & $631(27.6)$ \\
\hline Current drinker & $322(14.8)$ & Current drinker & $1,104(48,4)$ \\
\hline Missing & $60(2.8)$ & Missing & $137(6.0)$ \\
\hline Physical activity & & Physical activity & \\
\hline Yes & $323(14.8)$ & Yes & $1,343(58.8)$ \\
\hline \multicolumn{4}{|l|}{ Continued } \\
\hline
\end{tabular}




\begin{tabular}{|l|l|l|l|}
\hline China (CLHLS 2011) & $\mathbf{n}, \%$ & US (NHANES 2011-2014) & $\mathbf{n}, \%$ \\
\hline & $1,750(80.3)$ & No & $940(41.2)$ \\
\hline No & $107(4.9)$ & & \\
\hline Missing & & Sleep duration & \\
\hline Sleep duration & $383(17.6)$ & $<6 \mathrm{~h}$ & $286(12.5)$ \\
\hline$<6 \mathrm{~h}$ & $1,308(60.0)$ & $6-9 \mathrm{~h}$ & $1,872(82.0)$ \\
\hline $6-9 \mathrm{~h}$ & $475(21.8)$ & $>9 \mathrm{~h}$ & $120(5.3)$ \\
\hline$>9 \mathrm{~h}$ & $14(0.6)$ & Missing & $5(0.2)$ \\
\hline Missing & & BMI & \\
\hline BMI & $524(24.0)$ & Underweight $(0-18.5)$ & $38(1.7)$ \\
\hline Underweight $(0-18.5)$ & $1,243(57.0)$ & Normal $(18.5-25)$ & $606(26.5)$ \\
\hline Normal $(18.5-25)$ & $231(10.6)$ & Overweight $(25-30)$ & $808(35.4)$ \\
\hline Overweight $(25-30)$ & $67(3.1)$ & Obese (>=30) & $778(34.1)$ \\
\hline Obese $(>=30)$ & $115(5.3)$ & Missing & $53(2.3)$ \\
\hline Missing & & VD supplement & \\
\hline & & No & $1110(48.6)$ \\
\hline & & Yes & $1173(51.4)$ \\
\hline
\end{tabular}

Table 1. Baseline characteristics of the CLHLS and NHANES participants.

There was a large difference in the serum level of $25(\mathrm{OH}) \mathrm{D}$ between China and the US. The mean serum $25(\mathrm{OH}) \mathrm{D}$ level in China was much lower $(45.1 \mathrm{nmol} / \mathrm{L})$ than in the US $(83.5 \mathrm{nmol} / \mathrm{L})$ (Table 2). In China, serum $25(\mathrm{OH}) \mathrm{D}$ level decreased by age and was significantly higher in males $(\mathrm{p}<0.0001)$, while in the US, we saw an increasing trend with age and was significantly higher in females $(\mathrm{p}<0.0001)$. In both countries, serum $25(\mathrm{OH}) \mathrm{D}$ level differed by races. It was significantly higher in Han Chinese than in ethnic minorities in China $(\mathrm{P}=0.0357)$, and higher in non-Hispanic whites than other races $(\mathrm{p}<0.0001)$. In the US, higher serum 25(OH)D levels were associated with higher educational levels and family income. In China, older adults with formal education, and lowest tertile of household income had higher serum $25(\mathrm{OH}) \mathrm{D}$ level. In the US, serum 25(OH)D significantly decreased with the worse health condition $(\mathrm{p}<0.0001)$. In China, those who rated their health condition as "Very bad" had the lowest serum $25(\mathrm{OH}) \mathrm{D}$ concentration.

In China, current smokers and current drinkers had significantly higher serum $25(\mathrm{OH}) \mathrm{D}$ levels. In the US, serum 25(OH)D level was lower in current smokers, but was not affected by drinking behavior. In both countries, older adults having physical activities had significantly higher serum $25(\mathrm{OH}) \mathrm{D}$ level $(\mathrm{p}=0.0001$ and 0.001 , respectively). As for BMI, older adults with higher BMI had lower serum $25(\mathrm{OH}) \mathrm{D}$ level in the US $(\mathrm{p}<0.0001)$, but the similar trend did not appear in China.

There were different predictors of serum 25(OH)D between China and the US (Table 3). In China, participants who were aged 80 and over, females, ethnic minorities, with higher household income, bad at self-rated health, and current drinkers, had lower serum $25(\mathrm{OH}) \mathrm{D}$ level. In the US, participants who were younger than 70 , males, Mexican American, Mexican American, other Hispanics, had lower income, bad at self-rated health, did not have physical activity, were overweight, and obese, had lower serum $25(\mathrm{OH}) \mathrm{D}$ level. Similar findings were observed for the risk factors of vitamin D deficiency. Additionally, seasonal variation of serum $25(\mathrm{OH}) \mathrm{D}$ was significant in CLHLS.

\section{Discussion}

Vitamin D is an essential micronutrient to human health, but there is no consensus on the optimal level of vitamin D. The US National Academy of Medicine recommended a serum 25(OH)D level of $50 \mathrm{nmol} / \mathrm{L}^{5}$. However, the US Endocrine Society recommended a higher level of $75 \mathrm{nmol} / \mathrm{L}$ for optimal health benefits ${ }^{6}$. Additionally, the European Society for Clinical and Economic Aspects of Osteoporosis and Osteoarthritis (ESCEO) recommended a minimal 25(OH)D level of $75 \mathrm{nmol} / \mathrm{L}$ for fragile elderly subjects ${ }^{7}$. The European Menopause and Andropause Society (EMAS) recommended elderly people to achieve serum $25(\mathrm{OH}) \mathrm{D}$ levels of $75-225 \mathrm{nmol} / \mathrm{L}^{8}$. In China, the Osteoporosis Committee of China Gerontological Society adopted the same standard as the National Academy of Medicine, with vitamin D deficiency defined as less than $30 \mathrm{nmol} / \mathrm{L}$, insufficiency as 30-49.9 nmol/L, and sufficiency as more than $50 \mathrm{nmol} / \mathrm{L}^{9}$. However, all guidelines recommend or conclude that serum $25 \mathrm{OHD}$ concentrations below $25 \mathrm{nmol} / \mathrm{l}$ should be avoided in all subjects (of whatever age) ${ }^{10}$. In our study, 78 (3.4\%) NHANES participants and $668(30.6 \%)$ CLHLS participants were considered as severe vitamin D deficiency $(<30 \mathrm{nmol} / \mathrm{L})$. 398 NHANES participants (17.4\%) and 1,572 CLHLS participants (70.7\%) had a serum 25(OH)D level less than $50 \mathrm{nmol} / \mathrm{L}$, indicating vitamin D deficiency or insufficiency.

Our study found lower serum 25(OH)D concentration among Chinese participants than US participants (45.1 vs $83.5 \mathrm{nmol} / \mathrm{L}$ ), unexplained by possible confounding factors. The mean $25(\mathrm{OH}) \mathrm{D}$ concentration in our study was similar to some previous findings in China ${ }^{11-16}$. The mean serum $25(\mathrm{OH}) \mathrm{D}$ concentrations reported in prior studies in China are lower than our finding in the NHANES population, and also some prior studies in the US. Studies have been conducted to examine the $25(\mathrm{OH}) \mathrm{D}$ concentrations worldwide. In Europe, using the NIH-led international Vitamin D Standardized Program (VDSP) protocol, the mean 25(OH)D value of 5519 participants (mean age $=76.6$ ) from Iceland was $57.0 \mathrm{nmol} / \mathrm{L}$, and of 915 participants (mean age $=71.4$ ) from the Netherlands 


\begin{tabular}{|c|c|c|c|c|c|}
\hline \multicolumn{3}{|l|}{ China (CLHLS 2011) } & \multicolumn{3}{|l|}{ US (NHANES 2011-2014) } \\
\hline & Mean (SE) & P value & & Mean (SE) & P value \\
\hline Total & $45.1(0.7)$ & & Total & $83.5(1.0)$ & \\
\hline Month of blood draw & & $<0.0001$ & Season of blood draw & & $<0.0001$ \\
\hline May & $40.9(1.5)$ & & Summer & $85.6(1.1)$ & \\
\hline June & $43.1(0.9)$ & & Winter & $80.6(1.7)$ & \\
\hline July & $48.2(1.2)$ & & & & \\
\hline August & $66.6(5.7)$ & & & & \\
\hline September & $61.3(3.6)$ & & & & \\
\hline Age group & & $<0.0001$ & Age group & & 0.03 \\
\hline $65-69$ & $46.8(1.5)$ & & $65-69$ & $80.1(1.7)$ & \\
\hline $70-74$ & $45.9(1.3)$ & & $70-74$ & $84.9(1.6)$ & \\
\hline $75-79$ & $45.2(1.1)$ & & $75-79$ & $84.4(2.0)$ & \\
\hline $80+$ & $41.6(0.7)$ & & $80+$ & $85.9(1.6)$ & \\
\hline Gender & & $<0.0001$ & Gender & & $<0.0001$ \\
\hline Male & $50.4(1.0)$ & & Male & $78.2(0.9)$ & \\
\hline Female & $40.1(0.8)$ & & Female & $87.8(1.6)$ & \\
\hline Race & & 0.0357 & Race/Ethnicity & & $<0.0001$ \\
\hline Han Chinese & $45.7(0.7)$ & & Mexican American & $66.8(2.9)$ & \\
\hline Ethnic minorities & $40.7(1.7)$ & & Other Hispanics & $70.9(3.0)$ & \\
\hline Missing & $39.0(3.8)$ & & Non-Hispanic White & $86.1(1.0)$ & \\
\hline & & & Non-Hispanic Black & $69.7(1.6)$ & \\
\hline & & & Non-Hispanic Asian & $82.8(2.7)$ & \\
\hline & & & Other races & $86.8(2.3)$ & \\
\hline Education & & $<0.0001$ & Education & & 0.005 \\
\hline No formal education & $42.2(0.9)$ & & Less than 9th grade & $74.1(2.1)$ & \\
\hline Formal education & $47.4(1.0)$ & & $\begin{array}{l}\text { 9-11th grade (Includes 12th grade } \\
\text { with no diploma) }\end{array}$ & $82.8(2.2)$ & \\
\hline Missing & $44.6(2.7)$ & & $\begin{array}{l}\text { High school graduate/GED or } \\
\text { equivalent }\end{array}$ & $84.1(2.3)$ & \\
\hline & & & Some college or AA degree & $82.6(1.7)$ & \\
\hline & & & College graduate or above & $87.3(1.5)$ & \\
\hline & & & Missing & 87.4 & \\
\hline Marital Status & & $<0.0001$ & Marital Status & & 0.1 \\
\hline Married & $46.7(0.9)$ & & Married & $84.0(1.1)$ & \\
\hline Separated & $45.2(3.5)$ & & Separated & $71.7(3.6)$ & \\
\hline Divorced & $51.1(4.6)$ & & Divorced & $83.1(2.3)$ & \\
\hline Widowed & $41.7(0.9)$ & & Widowed & $83.3(2.1)$ & \\
\hline Never married & $54.3(9.1)$ & & Never married & $80.9(2.2)$ & \\
\hline Missing & $41.6(3.5)$ & & Missing & 50 & \\
\hline Household income & & 0.0073 & Income(PIR) & & $<0.0001$ \\
\hline Tertile1 (0-6,000 RMB) & $47.4(1.3)$ & & $0-1.85$ & $78.7(1.6)$ & \\
\hline Tertile2 (6,200-20,000 RMB) & $44.4(1.0)$ & & $1.86-3.50$ & $83.8(1.9)$ & \\
\hline Tertile3 (21,000- > 100,000 RMB) & $44.5(1.2)$ & & $>3.51$ & $88.1(1.5)$ & \\
\hline Missing & $39.4(2.1)$ & & Missing & $81.6(3.8)$ & \\
\hline Health condition & & $<0.0001$ & Health condition & & $<0.0001$ \\
\hline Very good & $45.5(2.3)$ & & Very good & $86.6(3.2)$ & \\
\hline Good & $45.9(1.1)$ & & Good & $89.3(2.4)$ & \\
\hline Fair & $45.4(0.9)$ & & Fair & $82.2(1.4)$ & \\
\hline $\mathrm{Bad}$ & $43.6(1.9)$ & & Bad & $77.5(2.0)$ & \\
\hline Very Bad & $32.7(3.8)$ & & Very Bad & 74.7 (4.5) & \\
\hline Missing & $36.9(3.0)$ & & Missing & $82.0(3.6)$ & \\
\hline Smoking status & & $<0.0001$ & Smoking status & & 0.03 \\
\hline Never smoker & $43.0(0.7)$ & & Never smoker & $84.4(1.3)$ & \\
\hline Former smoker & $49.4(2.1)$ & & Past smoker & $84.4(1.0)$ & \\
\hline Current smoker & $51.7(1.6)$ & & Current smoker & 74.6 (3.9) & \\
\hline Missing & $40.8(2.6)$ & & Missing & 41.8 & \\
\hline
\end{tabular}




\begin{tabular}{|c|c|c|c|c|c|}
\hline \multicolumn{3}{|l|}{ China (CLHLS 2011) } & \multicolumn{3}{|c|}{ US (NHANES 2011-2014) } \\
\hline & Mean (SE) & Pvalue & & Mean (SE) & Pvalue \\
\hline Drinking status & & $<0.0001$ & Drinking status & & 0.8 \\
\hline Never drinker & $43.2(0.7)$ & & Never drinker & $83.5(2.4)$ & \\
\hline Former drinker & $42.8(2.4)$ & & Past drinker & $84.1(1.9)$ & \\
\hline Current drinker & $55.8(1.9)$ & & Current drinker & $83.3(1.2)$ & \\
\hline Missing & $42.8(3.0)$ & & Missing & $82.9(3.8)$ & \\
\hline Physical activity & & $<0.0001$ & Physical activity & & 0.001 \\
\hline Yes & $46.0(1.4)$ & & Yes & $86.0(1.2)$ & \\
\hline No & $45.0(0.8)$ & & No & $79.4(1.5)$ & \\
\hline Missing & $44.2(3.3)$ & & & & \\
\hline Sleep duration & & $<0.0001$ & Sleep duration & & 0.5 \\
\hline$<6 \mathrm{~h}$ & $46.0(1.3)$ & & $<6 \mathrm{~h}$ & $79.6(2.7)$ & \\
\hline $6-9 \mathrm{~h}$ & $45.2(0.8)$ & & $6-9 \mathrm{~h}$ & $84.1(1.0)$ & \\
\hline$>9 \mathrm{~h}$ & $43.7(1.7)$ & & $>9 \mathrm{~h}$ & $80.1(4.7)$ & \\
\hline Missing & $41.4(7.9)$ & & Missing & 59.5 & \\
\hline BMI & & $<0.0001$ & BMI & & $<0.0001$ \\
\hline Underweight (0-18.5) & $46.4(1.8)$ & & Underweight $(0-18.5)$ & $91.9(5.7)$ & \\
\hline Normal (18.5-25) & $44.8(0.8)$ & & Normal (18.5-25) & $90.4(1.8)$ & \\
\hline Overweight (25-30) & $46.0(1.8)$ & & Overweight (25-30) & $83.3(1.5)$ & \\
\hline Obese $(>=30)$ & $44.7(3.1)$ & & Obese $(>=30)$ & $77.9(1.5)$ & \\
\hline \multirow[t]{4}{*}{ Missing } & $37.6(2.8)$ & & Missing & $84.1(2.7)$ & \\
\hline & & & VD supplement & & $<0.0001$ \\
\hline & & & No & $67.9(1.2)$ & \\
\hline & & & Yes & $94.7(1.0)$ & \\
\hline
\end{tabular}

Table 2. Serum 25(OH)D levels by baseline characteristics among CLHLS and NHANES participants.

was $64.7 \mathrm{nmol} / \mathrm{L}^{17}$. These results are higher than the value in China, but lower than the value in the US. Among these two cohorts, $8.4 \%$ and $4.6 \%$ were considered as severe deficiency ( $<30 \mathrm{nmol} / \mathrm{L}$ ), which is similar to our US population (3.4\%). In the Middle East countries, a study in Lebanon with 157 males and 286 females (mean age $=73$ ) found a mean $25(\mathrm{OH}) \mathrm{D}$ value of $25.7 \mathrm{nmol} / \mathrm{L}$. An Egypt study with elderly women (mean age $=76$ ) found a mean $25(\mathrm{OH}) \mathrm{D}$ value of $37 \mathrm{nmol} / \mathrm{L}^{18}$. These results are more comparable to the results in China ${ }^{19}$.

Furthermore, we found there was a difference in predictors related to serum $25(\mathrm{OH}) \mathrm{D}$ levels between China and the US. In both the US and China, older adults had lower serum 25(OH)D level, consistent with prior findings ${ }^{20,21}$. The decline of serum $25(\mathrm{OH}) \mathrm{D}$ level in the aging process is linked to reduction in the skin production of vitamin $\mathrm{D}$, calcium absorption of circulated $1,25(\mathrm{OH}) 2 \mathrm{D}$, and renal production of $1,25(\mathrm{OH}) 2 \mathrm{D}^{4}$. At the same time, vitamin D supplement intake helps increase the serum 25(OH)D level, especially for the older adults. We believe the different serum 25(OH)D level over age between China and the US may be explained by the much higher vitamin D usage in the US than in China ${ }^{20,22}$.

We found a small gender difference in serum $25(\mathrm{OH}) \mathrm{D}$ level, observed both in China and the US. In China, females had lower serum $25(\mathrm{OH}) \mathrm{D}$ level, while males had lower concentrations in the US. In the US, a study of 2007-2010 NHANES reported no significant gender difference among adults aged 65 years and older ${ }^{23}$, while another study of 1998-2004 NHANES showed that males had significantly higher serum 25(OH)D level than females $^{24}$. It may be possible that sun avoidance behavior was more prevalent in females, such as the use of sunscreen, protective clothes, and sunglasses ${ }^{25,26}$. Potential gender difference may also be caused by differences in hormone levels, lifestyle, and supplement usage ${ }^{27}$.

Income also affects serum $25(\mathrm{OH}) \mathrm{D}$ level. In the US, higher income was associated with higher serum $25(\mathrm{OH})$ $\mathrm{D}$ levels. The higher income group had more dietary supplement like vitamin $\mathrm{D}$, and also more diverse nutrient sources of vitamin $\mathrm{D}^{22}$. However, in China, the older adults with higher household income were more likely to have lower serum $25(\mathrm{OH}) \mathrm{D}$ level, and this finding is different from the majority findings in other countries ${ }^{28}$. Because of the rapid urbanizing process, those of higher socioeconomic status may be more likely to live in cities and areas with higher population density, and hence reduce outdoor sunlight exposure. In a study using the 20102013 China National Nutrition and Health Survey (CNNHS), older adults aged 60 years and older living in large cities had a higher risk of vitamin D inadequacy than those living in general rural areas ${ }^{20}$. Furthermore, high air pollution in cities could also act as a barrier to UV light, although this pathway has not been clearly elucidated ${ }^{29}$.

Both CLHLS and NHANES presented that the older adults who were very bad at self-rated health had lower serum $25(\mathrm{OH}) \mathrm{D}$ level. Lower $25(\mathrm{OH}) \mathrm{D}$ level is related to bad health conditions. Prior studies have found a strong association between $25(\mathrm{OH}) \mathrm{D}$ and several health conditions, including delirium, high blood pressure, and lower total testosterone $\mathrm{e}^{30-32}$, but there were also studies not supporting effects of 25(OH)D on diabetes, breast, prostate, and colorectal cancer ${ }^{33-35}$. On the other hand, bad health conditions may also lead to a reduced $25(\mathrm{OH}) \mathrm{D}$ level. For example, depressed individuals are often reluctant to engage in outdoor activities, and have reduced appetite, which can decrease $25(\mathrm{OH}) \mathrm{D}$ levels ${ }^{36}$. Hence, there might be a vicious cycle between lower vitamin $\mathrm{D}$ and bad health. 


\begin{tabular}{|c|c|c|c|c|c|c|c|c|c|}
\hline \multicolumn{5}{|l|}{ China (CLHLS 2011)* } & \multicolumn{5}{|l|}{ US (NHANES 2011-2014)** } \\
\hline Predictors & $\begin{array}{l}\text { Coefficient } \\
(95 \% \mathrm{CI})\end{array}$ & p value & OR $(95 \% \mathrm{CI})$ & p value & Predictors & $\begin{array}{l}\text { Coefficient } \\
\text { (95\%CI) }\end{array}$ & p value & OR $(95 \% \mathrm{CI})$ & p value \\
\hline Month of blood draw & & & & & Season of blood draw & & & & \\
\hline May & Ref & & Ref & & Winter & Ref & & Ref & \\
\hline June & $\begin{array}{l}5.19(2.06 \\
8.32)\end{array}$ & 0.001 & $\begin{array}{l}1.59(1.00 \\
2.53)\end{array}$ & 0.050 & Summer & $\begin{array}{l}3.58(-1.26 \\
8.43)\end{array}$ & 0.10 & $0.68(0.49,0.99)$ & 0.049 \\
\hline July & $\begin{array}{l}12.93(9.14 \\
16.71)\end{array}$ & $<0.001$ & $\begin{array}{l}3.16(1.90 \\
5.25)\end{array}$ & $<0.001$ & & & & & \\
\hline August & $\begin{array}{l}29.69(18.45 \\
40.93)\end{array}$ & $<0.001$ & $\begin{array}{l}19.14(6.68, \\
54.86)\end{array}$ & $<0.001$ & & & & & \\
\hline September & $\begin{array}{l}23.86(15.48, \\
32.23)\end{array}$ & $<0.001$ & $\begin{array}{l}8.35(2.57 \\
27.10)\end{array}$ & $<0.001$ & & & & & \\
\hline Age group & & & & & Age group & & & & \\
\hline 65-69 & Ref & & Ref & & $65-69$ & Ref & & Ref & \\
\hline $70-74$ & $\begin{array}{l}-0.73(-4.31 \\
2.85)\end{array}$ & 0.689 & $\begin{array}{l}1.12(0.68, \\
1.83)\end{array}$ & 0.653 & $70-74$ & $\begin{array}{l}4.59(0.57 \\
8.62)\end{array}$ & 0.03 & $0.61(0.38,0.99)$ & 0.046 \\
\hline $75-79$ & $\begin{array}{l}-1.90(-5.50 \\
1.70)\end{array}$ & 0.300 & $\begin{array}{l}0.80(0.47 \\
1.35)\end{array}$ & 0.402 & $75-79$ & $\begin{array}{l}5.19(0.13 \\
10.25)\end{array}$ & 0.04 & $0.62(0.37,1.05)$ & 0.07 \\
\hline $80+$ & $\begin{array}{l}-4.53(-8.05 \\
-1.02)\end{array}$ & 0.012 & $\begin{array}{l}0.66(0.40 \\
1.10)\end{array}$ & 0.113 & $80+$ & $\begin{array}{l}3.33(-1.36 \\
8.03)\end{array}$ & 0.2 & $0.77(0.50,1.21)$ & 0.3 \\
\hline Gender & & & & & Gender & & & & \\
\hline Male & Ref & & Ref & & Male & Ref & & Ref & \\
\hline Female & $\begin{array}{l}-7.16(-10.51 \\
-3.82)\end{array}$ & $<0.001$ & $\begin{array}{l}0.40(0.25 \\
0.64)\end{array}$ & $<0.001$ & Female & $\begin{array}{l}12.13(8.31 \\
15.94)\end{array}$ & $<0.0001$ & $1.05(0.68,1.64)$ & 0.8 \\
\hline Race & & & & & Race/Ethnicity & & & & \\
\hline Han Chinese & Ref & & Ref & & Mexican American & $\begin{array}{l}-9.57(-16.36 \\
-2.79)\end{array}$ & 0.01 & $2.92(1.59,5.38)$ & 0.0005 \\
\hline \multirow[t]{5}{*}{ Ethnic minorities } & $\begin{array}{l}-4.77(-8.72 \\
-0.82)\end{array}$ & 0.018 & $\begin{array}{l}0.48(0.24 \\
0.96)\end{array}$ & 0.037 & Other Hispanics & $\begin{array}{l}-11.39 \\
(-20.13 \\
-2.65)\end{array}$ & 0.01 & $2.19(1.02,4.69)$ & 0.04 \\
\hline & & & & & Non-Hispanic White & Ref & & Ref & \\
\hline & & & & & Non-Hispanic Black & $\begin{array}{l}-13.00 \\
(-16.78 \\
-9.23)\end{array}$ & $<0.0001$ & $3.43(2.35,5.03)$ & $<0.0001$ \\
\hline & & & & & Non-Hispanic Asian & $\begin{array}{l}-6.77(-12.83 \\
-0.71)\end{array}$ & 0.03 & $2.25(1.16,4.35)$ & 0.02 \\
\hline & & & & & Other races & $\begin{array}{l}8.13(-2.17 \\
18.43)\end{array}$ & 0.1 & $0.84(0.16,4.52)$ & 0.8 \\
\hline Education & & & & & Education & & & & \\
\hline No formal education & Ref & & Ref & & Less than 9th grade & Ref & & Ref & \\
\hline \multirow[t]{4}{*}{ Formal education } & $\begin{array}{l}0.05(-2.86, \\
2.97)\end{array}$ & 0.971 & $\begin{array}{l}1.05(0.70 \\
1.58)\end{array}$ & 0.813 & $\begin{array}{l}\text { 9-11th grade (Includes 12th } \\
\text { grade with no diploma) }\end{array}$ & $\begin{array}{l}2.48(-2.29 \\
-7.26)\end{array}$ & 0.30 & $1.20(0.67,2.13)$ & 0.5 \\
\hline & & & & & $\begin{array}{l}\text { High school graduate/GED or } \\
\text { equivalent }\end{array}$ & $\begin{array}{l}1.16(-5.23, \\
7.55)\end{array}$ & 0.70 & $1.58(0.83,3.03)$ & 0.2 \\
\hline & & & & & Some college or AA degree & $\begin{array}{l}-2.00(-8.06 \\
4.06)\end{array}$ & 0.50 & $1.02(0.55,1.90)$ & 0.9 \\
\hline & & & & & College graduate or above & $\begin{array}{l}0.26(-6.28 \\
6.81)\end{array}$ & 0.90 & $1.06(0.48,2.37)$ & 0.9 \\
\hline Marital Status & & & & & Marital Status & & & & \\
\hline Married & Ref & & Ref & & Married & Ref & & Ref & \\
\hline Separated & $\begin{array}{l}-2.14(-9.34 \\
5.07)\end{array}$ & 0.561 & $\begin{array}{l}0.57(0.15 \\
2.17)\end{array}$ & 0.414 & Separated & $\begin{array}{l}-6.57(-14.40, \\
1.26)\end{array}$ & 0.10 & $1.32(0.62,2.80)$ & 0.5 \\
\hline Divorced & $\begin{array}{l}3.32(-4.07 \\
10.71)\end{array}$ & 0.379 & $\begin{array}{l}1.72(0.22 \\
13.54)\end{array}$ & 0.606 & Divorced & $\begin{array}{l}-0.29(-6.10 \\
5.52)\end{array}$ & 0.90 & $1.32(0.67,2.62)$ & 0.4 \\
\hline Widowed & $\begin{array}{l}-0.49(-3.17 \\
2.18)\end{array}$ & 0.717 & $\begin{array}{l}0.88(0.59 \\
1.31)\end{array}$ & 0.529 & Widowed & $\begin{array}{l}-1.98(-6.55 \\
2.59)\end{array}$ & 0.40 & $1.44(0.86,2.41)$ & 0.2 \\
\hline Never married & $\begin{array}{l}-3.09(-18.49 \\
12.32)\end{array}$ & 0.694 & $\begin{array}{l}0.34(0.11 \\
1.09)\end{array}$ & 0.068 & Never married & $\begin{array}{l}-1.60(-7.20 \\
3.98)\end{array}$ & 0.60 & $0.98(0.59,1.64)$ & 0.9 \\
\hline Household income & & & & & Income(PIR) & & & & \\
\hline Tertile1 (0-6,000RMB) & Ref & & Ref & & $0-1.85$ & Ref & & Ref & \\
\hline Tertile2 (6,200-20,000 RMB) & $\begin{array}{l}-4.56(-7.44 \\
-1.69)\end{array}$ & 0.002 & $\begin{array}{l}0.63(0.41 \\
0.96)\end{array}$ & 0.032 & $1.86-3.50$ & $\begin{array}{l}2.96(-0.55 \\
6.48)\end{array}$ & 0.10 & $0.78(0.54,3.13)$ & 0.2 \\
\hline $\begin{array}{l}\text { Tertile3 } \\
(21,000->100,000 \mathrm{RMB})\end{array}$ & $\begin{array}{l}-4.48(-7.83 \\
-1.13)\end{array}$ & 0.009 & $\begin{array}{l}0.52(0.32 \\
0.83)\end{array}$ & 0.007 & $>3.51$ & $\begin{array}{l}7.02(1.25 \\
12.79)\end{array}$ & 0.02 & $0.55(0.30,0.99)$ & 0.048 \\
\hline Health condition & & & & & Health condition & & & & \\
\hline
\end{tabular}




\begin{tabular}{|c|c|c|c|c|c|c|c|c|c|}
\hline \multicolumn{5}{|l|}{ China (CLHLS 2011)* } & \multicolumn{5}{|c|}{ US (NHANES 2011-2014)*** } \\
\hline Predictors & \begin{tabular}{|l|} 
Coefficient \\
$(95 \% \mathrm{CI})$
\end{tabular} & p value & OR $(95 \% \mathrm{CI})$ & p value & Predictors & \begin{tabular}{|l|} 
Coefficient \\
$(95 \% \mathrm{CI})$
\end{tabular} & p value & OR $(95 \% \mathrm{CI})$ & p value \\
\hline Very good & $\begin{array}{l}-0.43(-5.48 \\
4.63)\end{array}$ & 0.869 & $\begin{array}{l}0.65(0.27, \\
1.57)\end{array}$ & 0.341 & Very good & $\begin{array}{l}1.45(-6.00, \\
8.91)\end{array}$ & 0.70 & $0.49(0.86,2.41)$ & 0.1 \\
\hline Good & \begin{tabular}{|l|}
$1.39(-1.32$, \\
$4.10)$
\end{tabular} & 0.315 & $\begin{array}{l}1.11(0.76, \\
1.64)\end{array}$ & 0.590 & Good & $\begin{array}{l}5.39(0.34, \\
10.43)\end{array}$ & 0.04 & $1.32(0.67,2.62)$ & 0.3 \\
\hline Fair & Ref & & Ref & & Fair & Ref & & Ref & \\
\hline $\mathrm{Bad}$ & $\begin{array}{l}-2.08(-6.12 \\
1.96)\end{array}$ & 0.313 & $\begin{array}{l}0.85(0.48 \\
1.48)\end{array}$ & 0.558 & Bad & $\begin{array}{l}-0.83(-4.63 \\
2.96)\end{array}$ & 0.70 & $1.44(0.86,2.41)$ & 0.4 \\
\hline Very Bad & $\begin{array}{l}-9.70(-14.10 \\
-5.31)\end{array}$ & $<0.001$ & $\begin{array}{l}0.29(0.076 \\
1.08)\end{array}$ & 0.065 & Very Bad & \begin{tabular}{|l|}
$-2.47(-12.94$ \\
$8.00)$
\end{tabular} & 0.04 & $0.98(0.59,1.64)$ & 0.5 \\
\hline Smoking status & & & & & Smoking status & & & & \\
\hline Never smoker & Ref & & Ref & & Never smoker & Ref & & Ref & \\
\hline Former smoker & $\begin{array}{l}2.78(-2.87, \\
8.42)\end{array}$ & 0.334 & $\begin{array}{l}1.23(0.60 \\
2.52)\end{array}$ & 0.577 & Former smoker & $\begin{array}{l}3.23(-0.34, \\
6.79)\end{array}$ & 0.07 & $1.03(0.74,1.44)$ & 0.9 \\
\hline Current smoker & $\begin{array}{l}0.68(-3.30, \\
4.65)\end{array}$ & 0.739 & $\begin{array}{l}1.31(0.83 \\
2.08)\end{array}$ & 0.246 & Current smoker & $\begin{array}{l}-3.91(-10.52 \\
2.71)\end{array}$ & 0.20 & $1.95(1.03,3.70)$ & 0.04 \\
\hline Drinking status & & & & & Drinking status & & & & \\
\hline Never drinker & Ref & & Ref & & Never drinker & Ref & & Ref & \\
\hline Former drinker & $\begin{array}{l}-2.82(-8.63, \\
2.99)\end{array}$ & 0.341 & $\begin{array}{l}0.89(0.44, \\
1.80)\end{array}$ & 0.742 & Former drinker & $\begin{array}{l}1.43(-4.60, \\
7.47)\end{array}$ & 0.60 & $0.98(0.63,1.53)$ & 0.9 \\
\hline Current drinker & $\begin{array}{l}8.70(4.34 \\
13.05)\end{array}$ & $<0.001$ & $\begin{array}{l}1.74(1.09 \\
2.79)\end{array}$ & 0.021 & Current drinker & $\begin{array}{l}-3.29(-7.89 \\
1.31)\end{array}$ & 0.20 & $1.40(0.88,2.23)$ & 0.2 \\
\hline Physical activity & & & & & Physical activity & & & & \\
\hline Yes & $\begin{array}{l}0.27(-3.04, \\
3.58)\end{array}$ & 0.872 & $\begin{array}{l}1.32(0.82 \\
2.11)\end{array}$ & 0.248 & Yes & $\begin{array}{l}4.87(1.61, \\
8.13)\end{array}$ & 0.01 & $0.63(0.43,0.94)$ & 0.02 \\
\hline No & Ref & & Ref & & No & Ref & & Ref & \\
\hline Sleep duration & & & & & Sleep duration & & & & \\
\hline$<6 \mathrm{~h}$ & $\begin{array}{l}-0.81(-4.02, \\
2.40)\end{array}$ & 0.621 & $\begin{array}{l}1.08(0.64, \\
1.84)\end{array}$ & 0.766 & $<6 \mathrm{~h}$ & $\begin{array}{l}-0.28(-6.96, \\
6.40)\end{array}$ & 0.90 & $0.92(0.45,1.85)$ & 0.8 \\
\hline 6-9h & Ref & & Ref & & $6-9 \mathrm{~h}$ & Ref & & Ref & \\
\hline$>9 \mathrm{~h}$ & $\begin{array}{l}-2.73(-6.39 \\
0.93)\end{array}$ & 0.144 & $\begin{array}{l}0.69(0.43, \\
1.12)\end{array}$ & 0.131 & $>9 \mathrm{~h}$ & \begin{tabular}{|l}
$-4.00(-13.74$ \\
$5.74)$
\end{tabular} & 0.4 & $2.51(1.46,4.28)$ & 0.0008 \\
\hline BMI & & & & & BMI & & & & \\
\hline Underweight $(0-18.5)$ & $\begin{array}{l}1.31(-2.16, \\
4.78)\end{array}$ & 0.460 & $\begin{array}{l}1.16(0.73, \\
1.84)\end{array}$ & 0.543 & Underweight (0-18.5) & $\begin{array}{l}-4.74(-23.03 \\
13.54)\end{array}$ & 0.6 & $0.84(0.08,8.47)$ & 0.9 \\
\hline $\begin{array}{l}\text { Normal } \\
(18.5-25)\end{array}$ & Ref & & Ref & & Normal (18.5-25) & Ref & & Ref & \\
\hline Overweight (25-30) & $\begin{array}{l}3.00(-0.56, \\
6.56)\end{array}$ & 0.099 & $\begin{array}{l}1.71(1.06, \\
2.75)\end{array}$ & 0.027 & Overweight (25-30) & $\begin{array}{l}-6.45(-10.10 \\
-2.81)\end{array}$ & 0.001 & $1.48(0.92,2.38)$ & 0.1 \\
\hline \multirow[t]{4}{*}{$\begin{array}{l}\text { Obese } \\
(>=30)\end{array}$} & $\begin{array}{l}3.10(-1.87, \\
8.06)\end{array}$ & 0.221 & $\begin{array}{l}2.13(0.94 \\
4.80)\end{array}$ & 0.069 & Obese $(>=30)$ & $\begin{array}{l}-11.96 \\
(-17.16 \\
-6.77)\end{array}$ & $<0.0001$ & $1.92(1.20,3.08)$ & 0.007 \\
\hline & & & & & vD supplement & & & & \\
\hline & & & & & No & Ref & & Ref & \\
\hline & & & & & Yes & \begin{tabular}{|l|}
$23.46(20.71-$ \\
$26.22)$
\end{tabular} & $<0.0001$ & $0.05(0.03-0.09)$ & $<0.0001$ \\
\hline
\end{tabular}

Table 3. Linear regression predicting serum 25(OH)D level and logistic regression predicting vitamin D deficiency in China and the US. *All regression models were adjusted for month of blood draw, age, gender, ethnicity, education, marital status, household income, health condition, smoking and drinking status, physical activity, sleep duration, and BMI in the CLHLS analysis. **All regression models were adjusted for season of sampling, age, gender, season, race/ethnicity, education, marital status, income, health condition, smoking and drinking status, physical activity, sleep duration, BMI, and vD supplement in the NHANES analysis.

Being physically inactive and overweight were risk factors of lower serum 25(OH)D level in the US, but not in China. Several studies reported the positive association between physical activity and serum $25(\mathrm{OH}) \mathrm{D}$ level ${ }^{37,38}$. Physical activity increases sun exposure, and prevents loss of muscle strength and mass, which are the essential determinants of serum 25(OH)D level ${ }^{38}$. However, evidence found that vitamin D insufficiency was still common among people who were highly physically active in Germany ${ }^{37}$. This probably partly contributes to the difference between China and the US. Additionally, the relationship between a higher BMI and a lower serum 25(OH)D level has been well studied ${ }^{39}$. Overweight or obese people may have lower dietary supplement intake, reduced cutaneous synthesis, decreased intestinal absorption, and need more vitamin D intake according to the volumetric dilution model. In China, the percentage of overweight or obese older adults was much lower, and we did not see an association between BMI and serum 25(OH)D level, possibly due to a smaller sample size. 
Our study used national representative samples and a diverse group of variables to assess. However, there were some limitations to our study as well. Firstly, different time of blood draw among the CLHLS and NHANES participants may bias our comparison analysis. In the CLHLS, the blood samples were collected from May to September, while in the NHANES, the samples were collected either in summer (May to October) or in winter (November to April). Serum 25(OH)D level is highly influenced by season due to sunlight availability, with higher concentrations in summer than in winter. Secondly, the measurement techniques of serum $25(\mathrm{OH}) \mathrm{D}$ used in the CLHLS differed from that in the NHANES. CLHLS applied enzyme-linked immunosorbent assay, while NHANES applied ultra-high performance liquid chromatography-tandem mass spectrometry (UHPLC-MS/ $\mathrm{MS})^{40}$. It was possible that different measurement techniques may contribute to a part of the difference in serum 25(OH)D between China and the US. However, it was unlikely to explain such a big difference in our study $(54.1 \mathrm{nmol} / \mathrm{L}$ in China vs. $83.5 \mathrm{nmol} / \mathrm{L}$ in the US). Thirdly, we had information vitamin D supplement use in the NHANES but not in the CLHLS. Since vitamin D supplement use helps increase the serum $25(\mathrm{OH}) \mathrm{D}$ level, we were not sure that how much difference in serum 25(OH)D concentrations between NHANES and CLHLS was contributed by vitamin D supplement use. Fourthly, some confounding factors such as comorbidities, time spent outdoors, and residential areas were recorded differently or unavailable, thus were not adjusted for to make the two datasets more comparable. However, we did adjust for the general health condition variable as a proxy for comorbidity which was consistent in both datasets. Lastly, our study used a cross-sectional design, which could neither infer any causal relationships nor show the difference in the trends of serum $25(\mathrm{OH}) \mathrm{D}$ over the years. There is a possibility that changes in $25(\mathrm{OH}) \mathrm{D}$ levels may in turn affect people's health conditions and behaviors. Further longitudinal studies could better inform the factors causally associated with $25(\mathrm{OH}) \mathrm{D}$.

Our findings demonstrated a large difference in 25(OH)D levels between US and Chinese older adults, which has implications for further research on whether the current clinical guideline is appropriate for people of different age, race, and country of residence. In addition to study design differences between CLHLS and NHANES, the factor of race and ethnicity cannot be ignored. Many studies have shown racial differences contrasting vitamin D status. In Australia, UK, and Canada, immigrants from Asia, Middle East, and Africa had significantly lower $25(\mathrm{OH}) \mathrm{D}$ levels compared to the white population ${ }^{41-43}$. In our study, in NHANES population, non-Hispanic Asians and non-Hispanic blacks also showed lower serum 25(OH)D level than non-Hispanic whites. African Americans generally have lower levels of vitamin $\mathrm{D}$ than their white counterparts due to skin pigmentation reducing vitamin $\mathrm{D}$ production ${ }^{44}$. The difference in skin color could contribute to their different $25(\mathrm{OH}) \mathrm{D}$ concentration. The difference in culture and tradition could also explain the differences in vitamin D status. Studies have found that the consumption of vitamin D-enriched food showed ethnic differences, which could lead to different $25(\mathrm{OH}) \mathrm{D}$ levels ${ }^{45,46}$. Furthermore, differences in population genetics can play a part in vitamin $\mathrm{D}$ synthesis and metabolism ${ }^{47}$. Studies have found that group-specific component gene (GC) polymorphisms were associated with 25(OH)D levels, and allele frequencies were different among geographic regions worldwide ${ }^{48}$. For example, the GC1S haplotype which is related to a higher level of $25(\mathrm{OH}) \mathrm{D}$ is found to have the maximum frequency in white population, while the GC1F haplotype which is associated with lower vitamin D-binding protein levels, is more likely to be carried by Asians ${ }^{49}$. Vitamin D-associated genes may present different allele frequency between cohorts. Genetic determinants of vitamin D production and metabolism may be the underlying reasons why there is a racial difference in health responses in clinical guidelines, and this warrants further investigation. Therefore, future studies and clinical guidelines should take race/ethnicity into consideration when examining $25(\mathrm{OH}) \mathrm{D}$ levels in different populations.

\section{Method}

Study population. We used data from CLHLS and NHANES to compare serum $25(\mathrm{OH}) \mathrm{D}$ concentrations among the older population, aged 65 years or older. Both CLHLS and NHANES collected data through in-person interviews and blood samples.

The CLHLS was designed to explore the determinants of healthy longevity among Chinese older adults. Established in 1998, the CLHLS recruited new participants and conducted follow-up surveys in 2000, 2002, 2005, 2008, 2011, 2014, and 2018. The CLHLS has collected extensive data on the determinants of health, including demographic characteristics, socioeconomic status, lifestyle, physical capacity, cognitive function, and psychological well-being. The CLHLS used a multistage, stratified cluster sampling, and recruited participants from 22 out of 31 provinces in China. 631 cities and counties were randomly selected as the sample sites, which represent about $85 \%$ of the Chinese population. More details about sampling design and weight could be found elsewhere ${ }^{50}$. Our study used the 2011 wave of CLHLS. CLHLS collected blood samples in eight longevity regions with a higher proportion of older people ${ }^{51}$. A total of 2,439 participants were surveyed in this wave. We excluded participants if they had missing values of $25(\mathrm{OH}) \mathrm{D}$ concentration $(\mathrm{n}=130)$, were younger than 65 years $(\mathrm{n}=84)$, and were missing weight variable $(n=45)$. We had 2,180 participants in CLHLS for final analysis.

NHANES is a nationally representative survey of the US non-institutionalized population, identified through a complex sampling design with oversampling of lower socioeconomic status and ethnicities minorities. Household interviews were conducted by trained personnel to collect information on health and socio-demographic characteristics. Standardized physical examinations were conducted and blood samples were also drawn in mobile examination centers. In the current analysis, data from the 2011-2012 and 2013-2014 waves were merged. Older adults who were 65 years or older were included as the study sample, which yielded a total of 2556 participants. Then, those who had missing values for serum $25(\mathrm{OH}) \mathrm{D}$ measurements were excluded from the analysis $(\mathrm{n}=273)$. The final sample consisted of 2283 older adults.

Vitamin D measurement. Serum 25-hydroxyvitamin D (25(OH)D) was considered as the best biomarker of vitamin D status since it indicates sources of both sun exposure and diet ${ }^{52}$. In CLHLS, 25(OH)D was assessed by an enzyme-linked immunosorbent assay (Immunodiagnostic Systems Limited, Bolton, UK). The 
inter- and intraassay coefficients of variation were less than $10 \%$ and less than $8 \%$, respectively ${ }^{53}$. In NHANES, ultra-high-performance liquid chromatography-tandem mass spectrometry (UHPLC-MS/MS) was utilized for the quantitative detection of $25(\mathrm{OH}) \mathrm{D}$. Details of the laboratory methodology, quality control protocol can be found in the Laboratory Method manuals ${ }^{54}$.

Covariates. We measured a number of covariates, including age, gender, race/ethnicity, marital status, education, household income, self-perceived health condition, smoking and drinking status, physical activity, sleep duration, body mass index (BMI), and time of blood draw. NHANES additionally measured vitamin D supplement. The phrasing of many questions in CLHLS and NHANES surveys were not identical, but were able to obtain measurements for each category. Missing values of covariates were reported separately (ranging from $0.1 \%-9 \%$ in both datasets).

In CLHLS, age was calculated as the difference between the interview dates and birth dates, verified through family members, genealogical records, ID cards, and household registration booklets. In NHANES, age was asked and recorded at the time of the screening. Individuals who were 80 years and over were topcoded as 80 years of age. In CLHLS, we coded ethnicity as Han Chinese and ethnic minorities, while in NHANES, race/ethnicity was categorized into Mexican American, other Hispanic, non-Hispanic White, non-Hispanic Black, non-Hispanic Asian, and other races. Years of schooling of CLHLS participants were divided into two groups: formal education $(>=1$ year education), and no formal education. In NHANES, educational level was divided into five categories: less than $9^{\text {th }}$ grade, $9-11^{\text {th }}$ grade, high school graduate/GED or equivalent, some college or AA degree, college graduate or above. In CLHLS, annual household income of one year before the interview year was recorded, and categorized into tertiles. In NHANES, poverty income ratio (PIR) for the household, which is the ratio of total family income to the poverty threshold for the year of the interview, was used to represent income level, and was divided into low income (0-1.85), middle income (1.86-3.50), and high income (>3.51). In both CLHLS and NHANES, we defined marital status as married, separated, divorced, widowed, and never married, and self-perceived health condition as very good, good, fair, bad, and very bad.

In CLHLS and NHANES, smoking and drinking behavior were coded as "never", "past" and "current" based on their answers in questionnaires. In CLHLS, we assessed the status of physical activity by the question of "whether exercise or not". In NHANES, physical activity was defined as having vigorous or moderate work/recreational activities, or walking or using bicycle in a typical week. In both CLHLS and NHANES, we divided sleep duration into $<6$ hours, 6 to 9 hours, and $>9$ hours. BMI is the body weight divided by the square of the body height (unit: $\mathrm{kg} / \mathrm{m}^{2}$ ). We used WHO standard of BMI in both CLHLS and NHANES, which defined a BMI of $<18.5 \mathrm{~kg} / \mathrm{m}^{2}$ as underweight, a BMI of $>=18.5$ to $<25 \mathrm{~kg} / \mathrm{m}^{2}$ as normal weight, a BMI of $>=25$ to $<30 \mathrm{~kg} / \mathrm{m}^{2}$ as overweight, and a BMI of $>=30$ as obese. Serum $25(\mathrm{OH}) \mathrm{D}$ has seasonal variation. The time of blood draw was recorded in months (May to September) in CLHLS, and in summer (May to October) or winter (November to April) in NHANES. Participants who reported taking vitamin D supplements 30 days prior to the survey were classified as having vitamin D supplements in NHANES.

Statistical analysis. We used SAS, version 9.4 (SAS Institute Inc., Cary, NC) for all analyses. We summarized participants' demographic and lifestyle characteristics using descriptive statistics. We reported the mean and SE (standard error) for continuous variables, sample size and proportion for categorical variables. Vitamin $\mathrm{D}$ status was dichotomized into non-deficiency $(>=50 \mathrm{nmol} / \mathrm{L})$ and deficiency $(<50 \mathrm{nmol} / \mathrm{L})$. We used linear regression and logistic regression to predict serum $25(\mathrm{OH}) \mathrm{D}$ concentration, adjusted for month/season of blood draw, age, gender, race/ethnicity, marital status, education, household income, self-perceived health condition, smoking and drinking status, physical activity, sleep duration, BMI, and vitamin D supplement. Weight was applied in the analysis to reflect the sampling design of the CLHLS and NHANES ${ }^{10}$. We calculated coefficients, Odds Ratios (ORs), and 95\% Confidence Intervals (CIs) to estimate the magnitude of predictors on serum $25(\mathrm{OH}) \mathrm{D}$ level. Results were considered significant at $\mathrm{p}<0.05$.

Ethical approval. NHANES was approved by the US Center for Disease Control and Prevention (CDC) National Center for Health Statistics Ethics Review Board, and CLHLS was approved by the Institutional Review Board (IRB) at Peking University and Duke University; participants in both studies gave informed consents. All methods were performed in accordance with the relevant guidelines and regulations.

\section{Data availability}

The datasets generated and/or analysed during the current study are available in the NHANES website. https:// wwwn.cdc.gov/nchs/nhanes/continuousnhanes/default.aspx?BeginYear=2005.

Received: 26 July 2019; Accepted: 9 December 2019;

Published online: 23 December 2019

\section{References}

1. Autier, P., Boniol, M., Pizot, C. \& Mullie, P. Vitamin D status and ill health: a systematic review. The lancet Diabetes \& endocrinology 2, 76-89 (2014).

2. Reid, I. R., Bolland, M. J. \& Grey, A. Effects of vitamin D supplements on bone mineral density: a systematic review and metaanalysis. The Lancet 383, 146-155 (2014).

3. Uday, S. \& Hogler, W. Nutritional Rickets and Osteomalacia in the Twenty-first Century: Revised Concepts, Public Health, and Prevention Strategies. Current osteoporosis reports 15, 293-302, https://doi.org/10.1007/s11914-017-0383-y (2017).

4. Gallagher, J. C. Vitamin D and aging. Endocrinology and Metabolism Clinics 42, 319-332 (2013).

5. Ross, A. C. et al. The 2011 report on dietary reference intakes for calcium and vitamin D from the Institute of Medicine: what clinicians need to know. The Journal of clinical endocrinology and metabolism 96, 53-58, https://doi.org/10.1210/jc.2010-2704 (2011). 
6. Holick, M. F. et al. Evaluation, treatment, and prevention of vitamin D deficiency: an Endocrine Society clinical practice guideline. The Journal of clinical endocrinology and metabolism 96, 1911-1930, https://doi.org/10.1210/jc.2011-0385 (2011).

7. Rizzoli, R. et al. Vitamin D supplementation in elderly or postmenopausal women: a 2013 update of the 2008 recommendations from the European Society for Clinical and Economic Aspects of Osteoporosis and Osteoarthritis (ESCEO). Current medical research and opinion 29, 305-313, https://doi.org/10.1185/03007995.2013.766162 (2013).

8. Perez-Lopez, F. R. et al. EMAS position statement: Vitamin D and postmenopausal health. Maturitas 71, 83-88, https://doi. org/10.1016/j.maturitas.2011.11.002 (2012).

9. Liao, X. P. et al. Application Guideline for Vitamin D and Bone Health in Adult Chinese(2014 Standard Edition) Chin. J Osteoporos 20, 1011-1030 (2014)

10. Bouillon, R. Comparative analysis of nutritional guidelines for vitamin D. Nature Reviews Endocrinology 13, 466, https://doi. org/10.1038/nrendo.2017.31 (2017)

11. Lu, Y. et al. The Relationship between Vitamin D and Type 2 Diabetes Is Intriguing: Glimpses from the Spect-China Study. 71, 195-202 (2017).

12. Wang, Y. et al. Vitamin D binding protein affects the correlation of $25(\mathrm{OH}) \mathrm{D}$ and frailty in the older men. 2014 (2014).

13. Aleteng, Q. et al. Optimal Vitamin D Status in a Middle-Aged and Elderly Population Residing in Shanghai. China. 23, 6001 (2017).

14. Zhen, D., Liu, L., Guan, C., Zhao, N. \& Tang, X. J. B. High prevalence of vitamin D deficiency among middle-aged and elderly individuals in northwestern China: its relationship to osteoporosis and lifestyle factors. 71, 1-6 (2015).

15. Chen, W. R. et al. Vitamin D, parathyroid hormone and risk factors for coronary artery disease in an elderly Chinese population. Journal of cardiovascular medicine (Hagerstown, Md.) 16, 59-68, https://doi.org/10.2459/jcm.0000000000000094 (2015).

16. Li, Y., Chen, C., Liu, H. L. \& Qian, G. Vitamin D, parathyroid hormone, and heart failure in a Chinese elderly population. Endocrine practice: official journal of the American College of Endocrinology and the American Association of Clinical Endocrinologists 21, 30-40, https://doi.org/10.4158/ep14123.Or (2015).

17. Cashman, K. D. et al. Vitamin D deficiency in Europe: pandemic? The American journal of clinical nutrition 103, 1033-1044, https:// doi.org/10.3945/ajcn.115.120873 (2016)

18. Lips, P. et al. Management of Endocrine Disease: Current vitamin D status in European and Middle East countries and strategies to prevent vitamin D deficiency; a position statement of the European Calcified Tissue Society. European journal of endocrinology, https://doi.org/10.1530/eje-18-0736 (2019).

19. Zhang, W., Stoecklin, E. \& Eggersdorfer, M. A glimpse of vitamin D status in Mainland China. Nutrition (Burbank, Los Angeles County, Calif.) 29, 953-957, https://doi.org/10.1016/j.nut.2013.01.010 (2013).

20. Chen, J. et al. Vitamin D status among the elderly Chinese population: A cross-sectional analysis of the 2010-2013 China national nutrition and health survey (CNNHS). Nutrition journal 16, 3 (2017).

21. Forrest, K. Y. \& Stuhldreher, W. L. Prevalence and correlates of vitamin D deficiency in US adults. Nutrition research 31, 48-54 (2011).

22. Gahche, J. J., Bailey, R. L., Potischman, N. \& Dwyer, J. T. Dietary supplement use was very high among older adults in the United States in 2011-2014. The Journal of nutrition 147, 1968-1976 (2017).

23. Jain, R. Variability in the levels of vitamin D by age, gender, and race/ethnicity: data from National Health and Nutrition Examination Survey 2007-2010. J Nutr Health Sci 3, 203 (2016).

24. Looker, A. C., Dawson-Hughes, B., Calvo, M., Gunter, E. \& Sahyoun, N. Serum 25-hydroxyvitamin D status of adolescents and adults in two seasonal subpopulations from NHANES III. Bone 30, 771-777 (2002).

25. Cheng, S. et al. Sun-exposure knowledge and protection behavior in a North Chinese population: a questionnaire-based study. Photodermatology, photoimmunology \& photomedicine 26, 177-181 (2010).

26. Holick, M. F. Vitamin D deficiency. New England Journal of Medicine 357, 266-281 (2007).

27. Qato, D. M. et al. Use of prescription and over-the-counter medications and dietary supplements among older adults in the United States. Jama 300, 2867-2878 (2008)

28. Mithal, A. et al. Global vitamin D status and determinants of hypovitaminosis D. J Osteoporosis International 20, 1807-1820, https:// doi.org/10.1007/s00198-009-0954-6 (2009).

29. Agarwal, K. S. et al. The impact of atmospheric pollution on vitamin D status of infants and toddlers in Delhi, India. Archives of disease in childhood 87, 111-113, https://doi.org/10.1136/adc.87.2.111 (2002).

30. Chen, C. et al. Causal Link Between Vitamin D and Total Testosterone in Men: A Mendelian Randomization Analysis. The Journal of clinical endocrinology and metabolism 104, 3148-3156, https://doi.org/10.1210/jc.2018-01874 (2019).

31. Kunutsor, S. K., Burgess, S., Munroe, P. B. \& Khan, H. Vitamin D and high blood pressure: causal association or epiphenomenon? European journal of epidemiology 29, 1-14, https://doi.org/10.1007/s10654-013-9874-z (2014).

32. Bowman, K. et al. Vitamin D levels and risk of delirium: A mendelian randomization study in the UK Biobank. Neurology 92, e1387-e1394, https://doi.org/10.1212/wnl.0000000000007136 (2019).

33. Wang, N. et al. Vitamin D, prediabetes and type 2 diabetes: bidirectional Mendelian randomization analysis. European journal of nutrition, https://doi.org/10.1007/s00394-019-01990-x (2019).

34. Jiang, X. et al. Circulating vitamin D concentrations and risk of breast and prostate cancer: a Mendelian randomization study. International journal of epidemiology, https://doi.org/10.1093/ije/dyy284 (2018).

35. He, Y. et al. Exploring causality in the association between circulating 25 -hydroxyvitamin D and colorectal cancer risk: a large Mendelian randomisation study. BMC medicine 16, 142, https://doi.org/10.1186/s12916-018-1119-2 (2018).

36. Parker, G. B., Brotchie, H. \& Graham, R. K. Vitamin D and depression. Journal of affective disorders 208, 56-61 (2017).

37. Klenk, J. et al. Objectively measured physical activity and vitamin D status in older people from Germany. J Epidemiol Community Health 69, 388-392 (2015).

38. Scott, D. et al. A prospective study of the associations between 25 -hydroxy-vitamin D, sarcopenia progression and physical activity in older adults. Clinical endocrinology 73, 581-587 (2010).

39. Vanlint, S. Vitamin D and obesity. Nutrients 5, 949-956 (2013).

40. Thienpont, L. M., Stepman, H. C. \& Vesper, H. W. Standardization of measurements of 25-hydroxyvitamin D3 and D2. Scandinavian Journal of Clinical and Laboratory Investigation 72, 41-49 (2012).

41. Preece, M. A. et al. Vitamin-D deficiency among Asian immigrants to Britain. Lancet (London, England) 1, 907-910, https://doi. org/10.1016/s0140-6736(73)91361-5 (1973).

42. Vieth, R., Cole, D. E., Hawker, G. A., Trang, H. M. \& Rubin, L. A. Wintertime vitamin D insufficiency is common in young Canadian women, and their vitamin D intake does not prevent it. European journal of clinical nutrition 55, 1091-1097, https://doi.org/10.1038/ sj.ejcn.1601275 (2001).

43. Brock, K., Wilkinson, M., Cook, R., Lee, S. \& Bermingham, M. Associations with Vitamin D deficiency in "at risk" Australians. The Journal of steroid biochemistry and molecular biology 89-90, 581-588, https://doi.org/10.1016/j.jsbmb.2004.03.073 (2004).

44. Harris, S. S. Vitamin D and African Americans. Journal of Nutrition 136, 1126-1129 (2006).

45. Holvik, K., Meyer, H. E., Haug, E. \& Brunvand, L. Prevalence and predictors of vitamin D deficiency in five immigrant groups living in Oslo, Norway: the Oslo Immigrant Health Study. European journal of clinical nutrition 59, 57-63, https://doi.org/10.1038/sj. ejcn.1602033 (2005).

46. van Dam, R. M. et al. Potentially modifiable determinants of vitamin D status in an older population in the Netherlands: the Hoorn Study. The American journal of clinical nutrition 85, 755-761, https://doi.org/10.1093/ajcn/85.3.755 (2007). 
47. Hansen, J. G. et al. Genetic and environmental factors are associated with serum 25 -hydroxyvitamin D concentrations in older African Americans. The Journal of nutrition 145, 799-805, https://doi.org/10.3945/jn.114.202093 (2015).

48. Elkum, N. et al. Vitamin D insufficiency in Arabs and South Asians positively associates with polymorphisms in GC and CYP2R1 genes. PloS one 9, e113102, https://doi.org/10.1371/journal.pone.0113102 (2014).

49. Nizamutdinov, I., Popov, I., Ilinsky, V. \& Rakitko, A. J. b. Allele frequency distribution of SNPs associated with levels of Vitamin D-binding protein and 25-hydroxyvitamin D. 564229 (2019).

50. Zeng, Y., Poston, D. L., Vlosky, D. A. \& Gu, D. Healthy longevity in China: Demographic, socioeconomic, and psychological dimensions. Vol. 20 (Springer Science \& Business Media, 2008).

51. Brasher, M. S., George, L. K., Shi, X., Yin, Z. \& Zeng, Y. Incorporating biomarkers into the study of socio-economic status and health among older adults in China. SSM-population health 3, 577-585 (2017).

52. Zerwekh, J. E. Blood biomarkers of vitamin D status. The American journal of clinical nutrition 87, 1087S-1091S (2008).

53. Chei, C. L. et al. Vitamin D Levels and Cognition in Elderly Adults in C hina. Journal of the American Geriatrics Society 62, 2125-2129 (2014).

54. CDC. Laboratory Procudure Manual (25-Hydroxyvitamin D3 3-epi-25-Hydroxyvitamin D3 25-Hydroxyvitamin D2). (National Center for Environmental Health, 2010).

\section{Acknowledgements}

This work was supported by the Bill \& Melinda Gates Foundation and the Kunshan Government Research Grant.

\section{Author contributions}

J.W., A.Z. and J.S.J. designed the study. J.W. and A.Z. had access to raw data and conducted statistical analysis. J.W., A.Z. and J.S.J. interpreted the results. All authors contributed to manuscript and approved of the final version.

\section{Competing interests}

The authors declare no competing interests.

\section{Additional information}

Correspondence and requests for materials should be addressed to J.S.J.

Reprints and permissions information is available at www.nature.com/reprints.

Publisher's note Springer Nature remains neutral with regard to jurisdictional claims in published maps and institutional affiliations.

(c) (i) Open Access This article is licensed under a Creative Commons Attribution 4.0 International License, which permits use, sharing, adaptation, distribution and reproduction in any medium or format, as long as you give appropriate credit to the original author(s) and the source, provide a link to the Creative Commons license, and indicate if changes were made. The images or other third party material in this article are included in the article's Creative Commons license, unless indicated otherwise in a credit line to the material. If material is not included in the article's Creative Commons license and your intended use is not permitted by statutory regulation or exceeds the permitted use, you will need to obtain permission directly from the copyright holder. To view a copy of this license, visit http://creativecommons.org/licenses/by/4.0/.

(C) The Author(s) 2019 